Mathematical Modelling and Analysis

Volume 17 Number 4, September 2012, 549-557

http://dx.doi.org/10.3846/13926292.2012.706656

(c) Vilnius Gediminas Technical University, 2012
Publisher: Taylor\&Francis and VGTU

http://www.tandfonline.com/TMMA

Print ISSN: 1392-6292

Online ISSN: 1648-3510

\title{
On I-Acceleration Convergence of Sequences of Fuzzy Real Numbers
}

\section{Amar Jyoti Dutta and Binod Chandra Tripathy}

\author{
Institute of Advanced Study in Science and Technology \\ Paschim Baragaon, Garchuk, Guwahati - 781035, Assam, India \\ E-mail(corresp.): amar_iasst@yahoo.co.in \\ E-mail: tripathybc@yahoo.com; tripathybc@rediffmail.com
}

Received August 11, 2011; revised June 6, 2012; published online September 1, 2012

\begin{abstract}
In this article we introduce the notion of ideal acceleration convergence of sequences of fuzzy real numbers. We have proved a decomposition theorem for ideal acceleration convergence of sequences as well as for subsequence transformations and studied different types of acceleration convergence of fuzzy real valued sequence.
\end{abstract}

Keywords: statistical convergence, statistical acceleration, limit inferior, limit superior.

AMS Subject Classification: 40A05; 40A35; $40 \mathrm{C} 05$.

\section{Introduction}

Faster convergence of sequences particularly the acceleration of convergence of sequence of partial sums of series via linear and nonlinear transformations are widely used in finding solutions of mathematical as well as different scientific and engineering problems. The problem of acceleration convergence often occurs in numerical analysis. To accelerate the convergence, the standard interpolation and extrapolation methods of numerical mathematics are quite helpful. It is useful to study about the acceleration of convergence methods, which transform a slowly converging sequence into a new sequence, converging to the same limit faster than the original sequence. Here we are interested in studying some properties for sequences of fuzzy real numbers in the process of acceleration convergence. The speed of convergence of sequences is of the central importance in the theory of sequence transformation.

A sequence transformation $T$ is a function $T:\left(x_{k}\right) \rightarrow\left(x_{k}^{*}\right)$ which maps a slowly convergent sequence to another sequence with better numerical properties. If $\lim _{k \rightarrow \infty} x_{k}=x$ and $\lim _{k \rightarrow \infty} x_{k}^{*}=x^{*}$ with $r_{n}$ and $r_{n}^{*}$ as the truncated errors. Then we have $x_{k}=x+r_{k}, x_{k}^{*}=x^{*}+r_{k}^{*}$. We say that the sequence $\left(x_{k}\right)$ converge more rapidly than the sequence $\left(x_{k}^{*}\right)$ if

$$
\lim _{k \rightarrow \infty} \frac{x_{k}^{*}-x^{*}}{x_{k}-x}=\lim _{k \rightarrow \infty} \frac{r_{k}^{*}}{r_{k}}=0 .
$$


Classically the convergence rate of a sequence is defined as follows.

Let $\left(x_{k}\right)$ be a real valued sequence with limit $x$. Then the convergence rate of $\left(x_{k}\right)$ is characterized by $\rho=\lim _{k \rightarrow \infty} \frac{x_{k+1}-x_{k}}{x_{k}-x}$, which closely resembles the ratio test in the theory of infinite series. If $0<|\rho|<1$, then $\left(x_{k}\right)$ is said to be linearly convergent. If $\rho=1$, then $\left(x_{k}\right)$ is said to be logarithmically convergent, if $\rho=0$ then $\left(x_{k}\right)$ is said to converge hyper-linearly and obviously $|\rho|>1$ stands for divergence of the sequence.

Typical examples of linearly convergent sequences are the partial sums of a geometric series (power series with a nonzero, but finite radius of convergence)

$$
s_{n}(z)=\sum_{k=0}^{\infty} z^{k}=\frac{1-z^{n+1}}{1-z}, \quad 0<|z|<1 .
$$

In contrast, the partial sums $\sum_{\nu=0}^{n}(\nu+1)^{-z}$ of the Dirichlet series for $\zeta(z)$ converge logarithmically.

Let $x=\left(x_{k}\right)$ and $y=\left(y_{k}\right)$ be two sequences with limits $\sigma$ and $\lambda$ respectively. Then the sequence $x$ is said to converge more rapidly than the sequence $y$, if $\lim _{k \rightarrow \infty} \frac{x_{k}-\sigma}{y_{k}-\lambda}=0$, provided $y_{k}-\lambda \neq 0$ for all $k \in N$ and it is denoted by $\left(x_{k}\right)<\left(y_{k}\right)$. If the sequences converge to the same limit $\lambda$, then $\left(x_{k}\right)<\left(y_{k}\right)$, if $\lim _{k \rightarrow \infty} \frac{x_{k}-\lambda}{y_{k}-\lambda}=0$. If the knowledge of the limit $\lambda$ is not known, sometimes it would be desirable to write it as

$$
\lim _{k \rightarrow \infty} \frac{x_{k+1}-x_{k}}{y_{k+1}-y_{k}}=\lim _{k \rightarrow \infty} \frac{\Delta x_{k}}{\Delta y_{k}}=0 .
$$

Let $x=\left(x_{n}\right)$ and $y=\left(y_{n}\right)$ be two sequences with limits $\sigma$ and $\lambda$ respectively. Then the sequence $x$ is said to be converge weakly faster than the sequence $y$ if there exists a constant $K$ such that $\left|x_{n}-\sigma\right| \leq K\left|y_{n}-\lambda\right|$, for all $n$.

The sequence transformation $T: x \rightarrow y$ is said to

(a) accelerate the convergence of the sequence $x$ if $y$ converges faster than $x$,

(b) weakly accelerate the convergence of the sequence $x$ if $y$ converges weakly faster than $x$.

At the initial stage, works on acceleration convergence was done by Smith and Ford [14], Keagy and Ford [11], Salzer [13], Dawson [9], Brezinski, Delahaye and Gesmain-Borne [7], Brezinski [6], Tripathy and Sen [27], Tripathy and Mahanta [24] and many others. In this article we have studied the $I$-acceleration convergence of sequences of fuzzy real numbers.

Different classes of sequence of fuzzy numbers have been introduced and their different properties have been investigated by Altinok, Colak and Et. [4], Altinok and Mursaleen [5], Altin, Mursaleen and Altinok [3], Colak, Altin and Mursaleen [8], Altin, Et., Basarir [1], Altin, Et. and Colak [2], Tripathy and Baruah [16, 17], Tripathy and Borgohain [15], Tripathy and Dutta [18, 19], Tripathy and Sarma $[25,26]$ and many others.

A fuzzy real number $X$ is a fuzzy set on $R$ i.e. a mapping $X: R \rightarrow I(=$ $[0,1])$, associating each real number $t$, with its grade of membership $X(t)$. 
The $\alpha$-level set of a fuzzy real number $X$ is denoted by $[X]^{\alpha}, 0<\alpha \leq 1$, where $[X]^{\alpha}=\{t \in R: X(t) \geq \alpha\}$. The 0-level set is the closure of strong 0-cut i.e. $\operatorname{cl}(\{t \in R: X(t)>0\})$.

A fuzzy real number $X$ is said to be upper-semi-continuous if for each $\varepsilon>0, X^{-1}([0, a+\varepsilon))$, for all $a \in I$ is open in the usual topology of $R$. If there exists $t \in R$ such that $X(t)=1$, then the fuzzy real number $X$ is called normal. A fuzzy real number $X$ is said to be convex, if $X(t) \geq$ $X(s) \wedge X(r)=\min (X(s), X(r))$, where $s<t<r$. The class of all upper semicontinuous, normal and convex fuzzy real numbers is denoted by $R(I)$. The set of real numbers $R$ can be embedded in $R(I)$, since each $r \in R$ can be regarded as a fuzzy number defined by

$$
\bar{r}(t)= \begin{cases}1, & \text { for } t=r \\ 0 & \text { otherwise }\end{cases}
$$

The additive identity and multiplicative identity of $R(I)$ are denoted by $\overline{0}$ and $\overline{1}$ respectively. The set $E^{F}$ of sequence of fuzzy real numbers is closed under the addition and scalar multiplication defined as follows:

For $\left(X_{k}\right),\left(Y_{k}\right) \in E^{F}, r \in R$

$$
\left(X_{k}\right)+\left(Y_{k}\right)=\left(X_{k}+Y_{k}\right) \in E^{F}, \quad r\left(X_{k}\right)=\left(r X_{k}\right) \in E^{F}
$$

where

$$
r X_{k}(t)= \begin{cases}X_{k}\left(r^{-1} t\right) & \text { if } r \neq 0 \\ \overline{0} & \text { if } r=0\end{cases}
$$

Let $X, Y \in R(I)$ and $\alpha$-level set be $X=\left[X_{1}^{\alpha}, X_{2}^{\alpha}\right]$ and $Y=\left[Y_{1}^{\alpha}, Y_{2}^{\alpha}\right], \alpha \in[0,1]$.

Some arithmetic operations on $R(I) \times R(I)$ in terms of $\alpha$-level sets are defined as follows:

$$
\begin{aligned}
& {[X+Y]^{\alpha}=\left[X_{1}^{\alpha}+Y_{1}^{\alpha}, X_{2}^{\alpha}+Y_{2}^{\alpha}\right], \quad[X-Y]^{\alpha}=\left[X_{1}^{\alpha}-Y_{2}^{\alpha}, X_{2}^{\alpha}-Y_{1}^{\alpha}\right],} \\
& {[k X]^{\alpha}= \begin{cases}{\left[k X_{1}^{\alpha}, k X_{2}^{\alpha}\right]} & \text { if } k \geq 0, \\
{\left[k X_{2}^{\alpha}, k X_{1}^{\alpha}\right]} & \text { otherwise. }\end{cases} } \\
& {[X \otimes Y]^{\alpha}=\left[\min _{i, j \in\{1,2\}} a_{i}^{\alpha} b_{j}^{\alpha}, \max _{i, j \in\{1,2\}} a_{i}^{\alpha} b_{j}^{\alpha}\right], \quad\left[X^{-1}\right]^{\alpha}=\left[\left(X_{2}^{\alpha}\right)^{-1},\left(X_{1}^{\alpha}\right)^{-1}\right]}
\end{aligned}
$$

Consider the metric $\bar{d}: R(I) \times R(I) \rightarrow R$ defined by

$$
\bar{d}(X, Y)=\sup _{0 \leq \alpha<1}\left\{\max \left(\left|X_{1}^{\alpha}-Y_{1}^{\alpha}\right|,\left|X_{2}^{\alpha}-Y_{2}^{\alpha}\right|\right)\right\}
$$

where $X=\left[X_{1}^{\alpha}, X_{2}^{\alpha}\right]$ and $Y=\left[Y_{1}^{\alpha}, Y_{2}^{\alpha}\right]$.

Clearly $R(I)$ is complete with respect to $\bar{d}$.

\section{Definitions and Background}

The notion of $I$-convergence was studied by Kostyrko, Macaj and Salat [12]. Later on the notion was further investigated from different aspects and linked 
with summability theory by Demrici [10], Tripathy and Hazarika [20, 21, 22, 23] and many others. If $X$ is a non-empty set, then a non-void class $I \subseteq 2^{X}$ is called an ideal if $I$ is additive (i.e. $A, B \in I \Rightarrow A \cup B \in I$ ) and hereditary (i.e. $A \in I$ and $B \subseteq A \Rightarrow B \in I$ ). An ideal $I \subseteq 2^{X}$ is said to be non-trivial if $I \neq 2^{X}$. A non empty family of sets $\Im \subseteq 2^{X}$ is a filter on $X$ if $\emptyset \notin \Im$ and for each $A, B \in \Im, A \cap B$ and $A \in \Im, B \supset A \Rightarrow B \in \Im$.

A sequence $\left(X_{n}\right)$ of fuzzy real numbers is said to be $I$-convergent to a fuzzy real number $L$ if for every $\varepsilon>0,\left\{n \in N_{0}: \bar{d}\left(X_{n}, L\right) \geq \varepsilon\right\} \in I$. We write it as, $I$ - $\lim X_{n}=L$. A subset $E$ of $N$ is said to have asymptotic density $\delta(E)$ if

$$
\delta(E)=\lim _{n \rightarrow \infty} \frac{1}{n} \sum_{k=1}^{n} \chi_{E}(k)
$$

exists, where $\chi_{E}$ is the characteristic function of $E$. Clearly all finite subsets of $N$ have zero asymptotic density and $\delta\left(E^{c}\right)=\delta(N \backslash E)=1-\delta(E)$.

A sequence $\left(x_{k}\right)$ is said to be statistically convergent to $L$ if for every $\varepsilon>0$, $\delta\left(k \in N:\left|x_{k}-L\right| \geq \varepsilon\right)=0$. We write $s t$-lim $x_{k}=L$ or $x_{k} \stackrel{s t}{\rightarrow} L$.

A subset $E$ of $N$ is said to have logarithmic density $d(E)$ if $d(E)=$ $\lim _{n \rightarrow \infty} \frac{1}{s_{n}} \sum_{k=1}^{n} \chi_{E}(k) / k$ exists, where $s_{n}=\sum_{k=1}^{n} 1 / k$, for all $n \in N$. Clearly all finite subsets of $N$ have zero logarithmic density and $d\left(E^{c}\right)=d(N \backslash E)=$ $1-d(E)$. Since $\sum_{k=1}^{n} 1 / k=\log n+\gamma+\mathcal{O}(1 / n)$, where $\gamma$ is the Euler constant, if $d(E)$ exists, then it is equal to $\lim _{n \rightarrow \infty} \frac{1}{\log n} \sum_{k=1}^{n} \chi_{E}(k) / k$.

Let $T=\left(t_{n k}\right)$ be a regular non-negative matrix. Then for $E \subset N$, if $d_{T}(E)=\lim _{n \rightarrow \infty} \sum_{k=1}^{\infty} t_{n k} \chi_{E}(k)$ exists, called the $T$-density of $E$. From the regularity of $T$ it follows that $\lim _{n \rightarrow \infty} \sum_{k=1}^{\infty} t_{n k}=1$ and from this and nonnegativity of $T$ it follows that $d_{T}(E) \in[0,1]$. Clearly the asymptotic density and logarithmic density can be obtained as the particular cases of $T$-density. If we consider $t_{n k}=1 / n$, for $k \leq n$ and $t_{n k}=0$ otherwise, then $d_{T}(E)=\delta(E)$. If we consider $t_{n k}=1 / k s_{n}$, for $k \leq n$ and $t_{n k}=0$ otherwise, then one will get $d_{T}(E)=d(E)$.

The uniform density of a subset $E$ of $N$ is defined as follows: For integers $t \geq 0$ and $s \geq 1$, let $E(t+1, s+1)=\operatorname{Card}\{n \in E: t+1 \leq n \leq t+s\}$. Let $\beta_{s}=\lim _{-i n f} f_{t \rightarrow \infty} E(t+1, t+s)$ and $\beta^{s}=\lim _{-\sup _{t \rightarrow \infty}} E(t+1, t+s)$. Then $\underline{u}(E)=\lim _{s \rightarrow \infty} \beta_{s} / s$ and $\bar{u}(E)=\lim _{s \rightarrow \infty} \beta^{s} / s$ exist. If $\underline{u}(E)=\bar{u}(E)$, then we say that the uniform density of $E$ exists and $u(E)=\underline{u}(E)=\bar{u}(E)$.

For a fuzzy real valued sequence $X=\left(X_{k}\right)$ consider the sets $A_{X}^{F}$ and $B_{X}^{F}$ defined by $A_{X}^{F}=\left\{\lambda \in R(I):\left\{k \in N: X_{k}>\lambda\right\} \notin I\right\}$ and $B_{X}^{F}=\{\mu \in$ $\left.R(I):\left\{k \in N: X_{k}<\mu\right\} \notin I\right\}$. For a sequence $X=\left(X_{k}\right)$ of fuzzy real numbers, the $I$-limit-superior and $I$-limit-inferior are defined as follows:

$$
I-\lim -\sup X= \begin{cases}\sup A_{X} & \text { for } A_{X} \neq \emptyset \\ -\bar{\infty} & \text { if } A_{X}=\emptyset\end{cases}
$$

and

$$
I \text {-lim-inf } X= \begin{cases}\sup B_{X} & \text { for } B_{X} \neq \emptyset \\ \bar{\infty} & \text { if } B_{X}=\emptyset\end{cases}
$$


For $I=I_{\delta}$ and $I=I_{d}$ it denotes the cases of statistical and logarithmic limit superior and limit inferior respectively.

We introduced the following definitions for fuzzy real-valued sequences.

Definition 1. The sequence $\left(X_{k}\right)$ converges to $L$, faster than the sequence $\left(Y_{k}\right)$ converges to $M$, written as $\left(X_{k}\right)<\left(Y_{k}\right)$ if, $\lim _{k \rightarrow \infty} \frac{\bar{d}\left(X_{k}, L\right)}{\bar{d}\left(Y_{k}, M\right)}=0$ provided $\bar{d}\left(Y_{k}, M\right) \neq 0$ for all $k \in N$.

Definition 2. Let $I$ - $\lim X_{k}=L$ and $I$ - $\lim Y_{k}=M$. Then the sequence $\left(X_{k}\right)$ converge $I$-faster than the sequence $\left(Y_{k}\right)$ written as $\left(X_{k}\right) \stackrel{I}{<}\left(Y_{k}\right)$ if $I-\lim _{k \rightarrow \infty} \frac{\bar{d}\left(X_{k}, L\right)}{\bar{d}\left(Y_{k}, M\right)}=0$.

Definition 3. Let $I$ - $\lim X_{k}=L$ and $I$ - $\lim Y_{k}=M$. The sequence $\left(X_{k}\right)$ converges to $L$ at the same rate as the sequence $\left(Y_{k}\right)$ converges to $M$, written as $\left(X_{k}\right) \stackrel{I}{\approx}\left(Y_{k}\right)$ if

$$
0<I \text {-lim-inf } \frac{\bar{d}\left(X_{k}, L\right)}{\bar{d}\left(Y_{k}, M\right)} \leq I \text {-lim-sup } \frac{\bar{d}\left(X_{k}, L\right)}{\bar{d}\left(Y_{k}, M\right)}<\infty \text {. }
$$

Definition 4. For the sequences $\left(X_{k}\right)$ and $\left(Y_{k}\right), X_{k} \stackrel{I}{=} Y_{k}$ for almost all $k$ related to $I$ (in short a.a.k.r.I), if $\left(n \in N: X_{k} \neq Y_{k}\right) \in I$.

Let $A=\left(a_{n k}\right)$ be an infinite matrix. For a sequence $X=\left(X_{k}\right)$, the $A$ transform of $X$ is defined as $A X=\left(A_{n} X\right)$, where $A_{n} X=\sum_{k=1}^{\infty} a_{n k} X_{k}$, for all $n \in N$.

The matrix $A=\left(a_{n k}\right)$ is said to accelerates the convergence of the sequence $X$, if $A X \stackrel{I}{<} X$. The acceleration field of $A$ is given by $\{X: A X \stackrel{I}{<} X\}$.

A subsequence $\left(X_{k(i)}\right)$ of $X$ can be represented as a regular matrix transformation $A$ times $X$ with $a_{i, n(i)}=1$ and $a_{p q}=0$ otherwise. It is clear that a subsequence can never converge slower than the original sequence. Some subsequences converge at the same rate and some converge faster than the sequence, but in every case $\lim _{i} \frac{\bar{d}\left(X_{k(i)}, \overline{0}\right)}{\bar{d}\left(X_{i}, \overline{0}\right)}<1$.

Throughout $\left(c_{0}^{I}\right)_{F}, c_{F}^{I}$ and $\left(m_{0}^{I}\right)_{F}$ will represent $I$-null, $I$-convergent and bounded $I$-null sequences of fuzzy real numbers respectively, $\left(S_{0}\right)^{I}$ and $\left(S_{0}^{I}\right)_{F}$ denote subsets of $\left(c_{0}^{I}\right)_{F}$ and $\left(m_{0}^{I}\right)_{F}$ respectively with non zero terms.

\section{Main Results}

Theorem 1. Let $\left(X_{k}\right),\left(Y_{k}\right) \in\left(S_{0}^{I}\right)_{F}$, then the following are equivalent.

(i) $\left(X_{k}\right) \stackrel{I}{<}\left(Y_{k}\right)$.

(ii) There exists $\left(X_{k}\right),\left(\dot{Y}_{k}\right) \in\left(S_{0}\right)_{F}$ such that $X_{k} \stackrel{I}{=} \dot{X}_{k}$ for a.a.k.r.I, $Y_{k} \stackrel{I}{=} \dot{Y}_{k}$ for a.a.k.r.I and $\left(X_{k}\right)<\left(Y_{k}\right)$.

(iii) There exists a subsets $K=\left\{k_{i}, i \in N\right\}$ of $N$ such that $K \in G$ and $\left(X_{k}\right)<\left(Y_{k}\right)$ on $K$. 
Proof. (i) $\Rightarrow$ (ii): Let $\left(X_{k}\right),\left(Y_{k}\right) \in\left(S_{0}^{I}\right)_{F}$ then there exist $A, B \in \Im$ such that $\lim _{k} X_{k}=\overline{0}$ over $A$ and $\lim _{k} Y_{k}=\overline{0}$ over $B$. Let $\left(X_{k}\right) \stackrel{I}{<}\left(Y_{k}\right)$ then there exist $C \subseteq \Im$ such that $\lim _{k} \frac{\bar{d}\left(X_{k}, \overline{0}\right)}{\bar{d}\left(Y_{k}, \overline{0}\right)}=0$ over $C$. Take $D=A \cap B \cap C$, clearly $D \subseteq \Im$. Consider the subsequences $\left(X_{k}\right),\left(\dot{Y}_{k}\right) \in\left(S_{0}\right)_{F}$ defined over $D$ such that $X_{k} \stackrel{I}{=} \dot{X}_{k}$ for a.a.k.r.I and $Y_{k} \stackrel{I}{=} \dot{Y}_{k}$ for a.a.k.r.I Clearly $\lim _{k} \frac{\bar{d}\left(\dot{X}_{k}, \overline{0}\right)}{\bar{d}\left(\dot{Y}_{k}, \overline{0}\right)}=0$, which imply $\left(\dot{X}_{k}\right)<\left(\dot{Y}_{k}\right)$.

(ii) $\Rightarrow$ (iii): Let $\left(\dot{X}_{k}\right),\left(\dot{Y}_{k}\right) \in\left(S_{0}\right)_{F}$, be such that $X_{k} \stackrel{I}{=} \dot{X}_{k}$ for a.a.k.r.I over $E \in \Im$ and $Y_{k}=\dot{Y}_{k}$ for a.a.k.r.I over $H \in \Im$ with $\left(\dot{X}_{k}\right)<\left(Y_{k}\right)$. Consider $K=E \cap H$ and $K=\left\{k_{i}: i \in N\right\}$, then $K \in \Im$. Clearly $X_{k_{i}} \stackrel{I}{=} X_{k_{i}}^{\prime} \in\left(S_{0}\right)_{F}$, $Y_{k_{i}} \stackrel{I}{=} \dot{Y}_{k} \in\left(S_{0}\right)_{F}$ and $\frac{\bar{d}\left(X_{k_{i}}, \overline{0}\right)}{\bar{d}\left(Y_{k_{i}}, \overline{0}\right)}=\frac{\bar{d}\left(X_{k_{i}}^{\prime}, \overline{0}\right)}{\bar{d}\left(Y_{k_{i}}^{\prime}, \overline{0}\right)}=0$ as $i \rightarrow \infty$. Thus $\left(X_{k_{i}}^{\prime}\right)<\left(Y_{k_{i}}\right)$.

(iii) $\Rightarrow$ (i): For $K=\left\{k_{i}, i \in N\right\}$ of $N, K \subseteq \Im$ and $\left(X_{k_{i}}^{\prime}\right)<\left(Y_{k_{i}}^{\prime}\right)$. This imply $\frac{\bar{d}\left(X_{k_{i}}^{\prime}, \overline{0}\right)}{\bar{d}\left(Y_{k_{i}}^{\prime}, \overline{0}\right)}=0$ as $i \rightarrow \infty$. Therefore $I$ - $\lim _{k \rightarrow \infty} \frac{\bar{d}\left(X_{k}, \overline{0}\right)}{\bar{d}\left(Y_{k}, \overline{0}\right)}=0$, over $K$. Hence $\left(X_{k}\right) \stackrel{I}{<}\left(Y_{k}\right)$.

Theorem 2. Let $\left(X_{k}\right)$ and $\left(Y_{k}\right)$ be sequences of fuzzy real numbers such that $\left(X_{k}\right) \stackrel{I}{<}\left(Y_{k}\right)$ and $\left(Y_{k}\right) \stackrel{I}{\approx}\left(Z_{k}\right)$. Then $\left(X_{k}\right) \stackrel{I}{<}\left(Z_{k}\right)$.

Proof. Consider the fuzzy real valued sequences $\left(X_{k}\right),\left(Y_{k}\right)$ and $\left(Z_{k}\right)$. Let $\left(X_{k}\right) \stackrel{I}{<}\left(Y_{k}\right)$ and $\left(Y_{k}\right) \stackrel{I}{\approx}\left(Z_{k}\right)$. We get $I-\lim \frac{\bar{d}\left(X_{k}, \overline{0}\right)}{\bar{d}\left(Y_{k}, \overline{0}\right)}=0$ and $0<$ $I$-lim-inf $\frac{\bar{d}\left(X_{k}, \overline{0}\right)}{\bar{d}\left(Y_{k}, \overline{0}\right)}<I$-lim-sup $\frac{\bar{d}\left(X_{k}, \overline{0}\right)}{\bar{d}\left(Y_{k}, \overline{0}\right)}<\infty$. Now we can write

$$
\begin{aligned}
& \left(I-\lim _{k \rightarrow \infty} \frac{\bar{d}\left(X_{k}, \overline{0}\right)}{\bar{d}\left(Y_{k}, \overline{0}\right)}\right)\left(I-\lim _{k \rightarrow \infty} \frac{\bar{d}\left(Y_{k}, \overline{0}\right)}{\bar{d}\left(Z_{k}, \overline{0}\right)}\right)=0 \\
& \quad \Rightarrow \quad I-\left(\lim _{k \rightarrow \infty} \frac{\bar{d}\left(X_{k}, \overline{0}\right)}{\bar{d}\left(Y_{k}, \overline{0}\right)} \cdot \frac{\bar{d}\left(Y_{k}, \overline{0}\right)}{\bar{d}\left(Z_{k}, \overline{0}\right)}\right)=0 \quad \Rightarrow \quad I-\left(\lim _{k \rightarrow \infty} \frac{\bar{d}\left(X_{k}, \overline{0}\right)}{\bar{d}\left(Z_{k}, \overline{0}\right)}\right)=0 .
\end{aligned}
$$

This implies that $\left(X_{k}\right) \stackrel{I}{<}\left(Z_{k}\right)$.

Theorem 3. Let $\left(X_{k}\right),\left(Y_{k}\right) \in\left(S_{0}^{I}\right)_{F}$ be such that $\left(X_{k}\right) \stackrel{I}{<}\left(Y_{k}\right)$. Then there exist a sequence $\left(Z_{k}\right) \in\left(S_{0}^{I}\right)_{F}$ such that $\left(X_{k}\right) \stackrel{I}{<}\left(Z_{k}\right) \stackrel{I}{<}\left(Y_{k}\right)$.

Proof. Consider the sequences $\left(X_{k}\right),\left(Y_{k}\right)$ and $\left(Z_{k}\right)$ taken from $\left(S_{0}^{I}\right)_{F}$ such that $\left(X_{k}\right) \stackrel{I}{<}\left(Y_{k}\right)$ and define $\left(Z_{k}\right)$ as $Z_{k}=\left(X_{k}\right)^{2 / 3} \cdot\left(Y_{k}\right)^{1 / 3}$. It is clear that $\left(Z_{k}\right) \in\left(S_{0}^{I}\right)_{F}$. We observe that

$$
\frac{\bar{d}\left(X_{k}, \overline{0}\right)}{\bar{d}\left(Z_{k}, \overline{0}\right)}=\frac{\bar{d}\left(X_{k}, \overline{0}\right)}{\left(\bar{d}\left(X_{k}, \overline{0}\right)\right)^{2 / 3} \cdot\left(\bar{d}\left(Y_{k}, \overline{0}\right)\right)^{1 / 3}}=\left(\frac{\bar{d}\left(X_{k}, \overline{0}\right)}{\bar{d}\left(Y_{k}, \overline{0}\right)}\right)^{1 / 3}
$$

and

$$
\frac{\bar{d}\left(Z_{k}, \overline{0}\right)}{\bar{d}\left(Y_{k}, \overline{0}\right)}=\frac{\left(\bar{d}\left(X_{k}, \overline{0}\right)\right)^{2 / 3} \cdot\left(\bar{d}\left(Y_{k}, \overline{0}\right)\right)^{1 / 3}}{\bar{d}\left(Y_{k}, \overline{0}\right)}=\left(\frac{\bar{d}\left(X_{k}, \overline{0}\right)}{\bar{d}\left(Y_{k}, \overline{0}\right)}\right)^{2 / 3}
$$


Therefore it follows that $I-\lim _{k \rightarrow \infty} \frac{\bar{d}\left(X_{k}, \overline{0}\right)}{\bar{d}\left(Y_{k}, \overline{0}\right)}=0$ and $I$ - $\lim _{k \rightarrow \infty} \frac{\bar{d}\left(Z_{k}, \overline{0}\right)}{\bar{d}\left(Y_{k}, \overline{0}\right)}=0$. Thus we conclude that $\left(X_{k}\right) \stackrel{I}{<}\left(Z_{k}\right) \stackrel{I}{<}\left(Y_{k}\right)$.

Theorem 4. If $A$ is a subsequence transformation that accelerates $\left(X_{k}\right)$ with respect to $I$ and $\left(Y_{k}\right)$ is a sequence in $\left(S_{0}^{I}\right)_{F}$ such that $\left(X_{k}\right) \stackrel{I}{=}\left(Y_{k}\right)$. Then $A$ accelerates $\left(Y_{k}\right)$.

Proof. Let $\left(X_{k}\right) \in\left(S_{0}^{I}\right)_{F}$ then there exists $E \subset N$ with $E \in \Im$ such that $\lim _{k} X_{k}=\overline{0}$ over $E$. Consider $\left(X_{k(i)}\right) \in\left(S_{0}^{I}\right)_{F}$, then there exists a $B \subset N$ with $B \in \Im$ such that $\lim _{k} X_{k(i)}=\overline{0}$ over $B$. Since $A$ accelerate $\left(X_{k}\right)$ with respect to $I$, so it imply $I-\lim \frac{\bar{d}\left(X_{k(i)}, \overline{0}\right)}{\bar{d}\left(X_{i}, \overline{0}\right)}=0$. Therefore there exists a subset $D$ of $N$ with $D \in \Im$ such that $\lim _{i} \frac{\bar{d}\left(X_{k(i)}, \overline{0}\right)}{\bar{d}\left(X_{i}, \overline{0}\right)}=0$ over $D$. Consider $\left(Y_{k}\right) \in\left(S_{0}^{I}\right)_{F}$ such that $\left(X_{k}\right) \stackrel{I}{=}\left(Y_{k}\right)$. We can write

$$
\frac{\bar{d}\left(Y_{k(i)}, \overline{0}\right)}{\bar{d}\left(Y_{i}, \overline{0}\right)}=\frac{\bar{d}\left(Y_{k(i)}, \overline{0}\right)}{\bar{d}\left(X_{k(i)}, \overline{0}\right)} \cdot \frac{\bar{d}\left(X_{i}, \overline{0}\right)}{\bar{d}\left(Y_{i}, \overline{0}\right)} \cdot \frac{\bar{d}\left(X_{k(i)}, \overline{0}\right)}{\bar{d}\left(X_{i}, \overline{0}\right)}
$$

Clearly $I-\lim \frac{\bar{d}\left(Y_{k(i)}, \overline{0}\right)}{d\left(Y_{i}, \overline{0}\right)}=0$. This completes the proof.

Theorem 5. If $A$ is a subsequence transformation that accelerates $\left(X_{k}\right) \in$ $\left(S_{0}^{I}\right)_{F}$ such that $A X \stackrel{I}{<} X$, then there exist a sequence $\left(Y_{k}\right) \in\left(S_{0}^{I}\right)_{F}$ such that $\left(X_{k}\right) \stackrel{I}{<}\left(Y_{k}\right)$ and $A$ accelerates $\left(Y_{k}\right)$.

Proof. Easy, in view of the proof of Theorem 4, so it is omitted.

Theorem 6. Let $\left(X_{k}\right) \in\left(S_{0}^{I}\right)_{F}$ and $A$ be a subsequence transformation. Then the following are equivalent.

(i) A accelerate $\left(X_{k}\right)$ with respect to $I$.

(ii) There exist a subsets $D=\left\{k_{i} ; i \in N\right\}$ of $N$ such that $D \in \Im$ and $A$ accelerates $\left(X_{n_{k}}\right)$.

Proof. (i) $\Rightarrow$ (ii): Let $\left(X_{k}\right) \in\left(S_{0}^{I}\right)_{F}$, then there exist a subset $B$ of $N$ with $B \in \Im$ such that $\lim _{k} X_{k}=\overline{0}$, over $B$. Since $A$ accelerate $\left(X_{k}\right)$ with respect to $I$, then $\left(X_{n_{k}}\right) \in\left(S_{0}^{I}\right)_{F}$ so that there exists a subset $C$ of $N$ with $C \in \Im$ such that $\lim _{k} X_{n_{k}}=\overline{0}$ and $I-\lim \frac{\bar{d}\left(X_{n_{k}}, \overline{0}\right)}{\bar{d}\left(X_{k}, \overline{0}\right)}=0$. Let $D=B \cap C$, then clearly $D \in \Im$. Since $D \subset N$, we can take $D=\left\{k_{i} ; i \in N\right\}$.

(ii) $\Rightarrow$ (i): The proof follows directly from the definition.

\section{References}

[1] Y. Altin, M. Et. and M. Basarir. On some generalized difference sequences of fuzzy numbers. Kuwait J. Sci. Engrg., 34(1A):1-14, 2007. 
[2] Y. Altin, M. Et. and R. Colak. Lacunary statistical and lacunary strongly convergence of generalized difference sequences of fuzzy numbers. Comput. Math. Appl., 52(6-7):1011-1020, 2006. http://dx.doi.org/10.1016/j.camwa.2006.03.025.

[3] Y. Altin, M. Mursaleen and H. Altinok. Statistical summability $(C, 1)$ for sequences of fuzzy real numbers and a Tauberian theorem. J. Intelligent Fuzzy Syst., 21(6):379-384, 2010.

[4] H. Altinok, R. Colak and M. Et. $\lambda$-Difference sequence spaces of fuzzy numbers. Fuzzy Sets Sys., 160(21):3128-3139, 2009. http://dx.doi.org/10.1016/j.fss.2009.06.002.

[5] H. Altinok and M. Mursaleen. $\Delta$-Statistical boundedness for sequences of fuzzy numbers. Taiwanese J. Math., 15(5):2081-2093, 2011.

[6] C. Brezinski. Review of methods to accelerate the convergence of sequences. Rend. Math., 7(6):303-316, 1974.

[7] C. Brezinski, J.P. Delahage and B. Gemain-Bonne. Convergence acceleration by extraction of linear subsequences. SIAM J. Numer. Anal., 20:1099-1105, 1983. http://dx.doi.org/10.1137/0720079.

[8] R. Colak, Y. Altin and M. Mursaleen. On some sets of difference sequences of fuzzy numbers. Soft Comput., 15(4):787-793, 2011. http://dx.doi.org/10.1007/s00500-010-0633-8.

[9] D.F. Dawson. Matrix summability over certain classes of sequences ordered with respect to rate of convergence. Pacific J. Math., 24(1):51-56, 1968.

[10] K. Demirci. I-limit superior and limit inferior. Math. Commun., 6:165-172, 2001.

[11] T.A. Keagy and W.F. Ford. Acceleration by subsequence transformation. Pacific J. Math., 132(2):357-362, 1988.

[12] P. Kostyrko, M. Macaj and T. Salat. I-Convergence. Real Anal. Exchange, 26(2):669-686, 2000.

[13] H.E. Salzer. A simple method for summing certain slowly convergent series. $J$. Math. Phys., 33:356-359, 1955.

[14] D.A. Smith and W.F. Ford. Acceleration of linear and logarithmic convergence. SIAM J. Numer. Anal., 16(2):223-240, 1979. http://dx.doi.org/10.1137/0716017.

[15] B.C. Tripathy and S. Bargohain. The sequence space $m\left(M, \phi, \Delta_{m}^{n}, p\right)^{F}$. Math. Model. Anal., 13(4):577-586, 2008. http://dx.doi.org/10.3846/1392-6292.2008.13.577-586.

[16] B.C. Tripathy and A. Baruah. New type of difference sequence spaces of fuzzy real numbers. Math. Model. Anal., 14(3):391-397, 2009. http://dx.doi.org/10.3846/1392-6292.2009.14.391-397.

[17] B.C. Tripathy and A. Baruah. Lacunary statistically convergent and lacunary strongly convergent generalized difference sequences of fuzzy real numbers. Kyungpook Math. J., 50:565-574, 2010.

[18] B.C. Tripathy and A.J. Dutta. On fuzzy real-valued double sequence spaces ${ }_{2} \ell_{F}^{p}$. Math. Comput. Modelling, 46:1294-1299, 2007. http://dx.doi.org/10.1016/j.mcm.2007.01.006.

[19] B.C. Tripathy and A.J. Dutta. Fuzzy real valued bounded variation double sequence space. Comput. Math. Appl., 59(2):1031-1037, 2010. http://dx.doi.org/10.1016/j.camwa.2009.09.006. 
[20] B.C. Tripathy and B. Hazarika. I-convergent sequence spaces associated with multiplier sequence spaces. Math. Inequal. Appl., 11(3):543-548, 2008.

[21] B.C. Tripathy and B. Hazarika. Paranormed $I$-convergent sequences spaces. Math. Slovaca, 59(4):485-494, 2009.

http://dx.doi.org/10.2478/s12175-009-0141-4.

[22] B.C. Tripathy and B. Hazarika. I-convergent sequences spaces defined by Orlicz function. Acta Math. Appl. Sin. Engl. Ser., 27(1):149-154, 2011.

http://dx.doi.org/10.1007/s10255-011-0048-z.

[23] B.C. Tripathy and B. Hazarika. $I$-monotonic and $I$-convergent sequences. Kyungpook Math. J., 51(2):233-239, 2011. http://dx.doi.org/10.5666/KMJ.2011.51.2.233.

[24] B.C. Tripathy and S. Mahanta. On $I$-acceleration convergence of sequences. $J$. Franklin Inst., 347:591-598, 2010. http://dx.doi.org/10.1016/j.jfranklin.2010.02.001.

[25] B.C. Tripathy and B. Sarma. Sequence spaces of fuzzy real numbers defined by Orlicz functions. Math. Slovaca, 58(5):621-628, 2008. http://dx.doi.org/10.2478/s12175-008-0097-9.

[26] B.C. Tripathy and B. Sarma. Double sequence spaces of fuzzy numbers defined by Orlicz function. Acta Math. Sci., 31B(1):134-140, 2011. http://dx.doi.org/10.1016/S0252-9602(11)60215-4.

[27] B.C. Tripathy and M. Sen. A note on rate of convergence of sequences and density of subsets of natural numbers. Ital. J. Pure Appl. Math, 17:151-158, 2005 . 